Luis Javier Sanz, Isabel Elustondo, Magdalena Valverde,

José F. Montilla, Marta Miralles

\title{
Salud mental e inmigración: adhesión al tratamiento ambulatorio
}

RESUMEN: La inmigración pone a prueba las capacidades y recursos de los sujetos para afrontar la dura tarea de asentamiento e integración en un país desconocido y lejano al propio.

PALABRAS CLAVE: Inmigración, adhesión, salud mental.
SUMMARY: Immigrants develop their capacities and resources to confront the hard task of establishment and integrating in a foreign country.

KEY WORDS: Migration, adhesion, mental health.

\section{Introducción}

Según datos de la Delegación del Gobierno para la Extranjería e Inmigración, en España, el número de extranjeros con tarjeta o autorización de residencia en vigor a 31 de Diciembre de 2005 es de 2.738.932. Del total, el $36 \%$ (986.178 personas) tienen origen iberoamericano, el 23,7\% (649.251 personas) provienen de África y el 20,78\% (569.284 personas) de la Europa Comunitaria, siendo estas tres las procedencias mayoritarias. Esto supone un incremento en la cifra total de dicha población del 38,52\% con respecto al año anterior (1). Estas cifras no reflejan la realidad total ya que si atendemos a datos del Padrón Municipal publicados por el Instituto Nacional de Estadística a 1 de enero de 2005, la población extranjera empadronada en España alcanzaba la cifra de 4.391 .484 (9.95\% del total de la población) lo que indica la existencia de más de un millón y medio de personas en situación irregular, sin contar aquellas que no están en ninguno de los registros, cifra que desconocemos (2). En el caso concreto del municipio de Getafe la situación no es distinta (Tabla 1): en tan sólo cinco años la población inmigrante se ha multiplicado por cinco, de 3.252 inmigrantes el 1 de enero de 2000 se ha pasado a una cifra de 15.936 a fecha de 1 de enero de 2004 , lo que supone un $9,90 \%$ de población total del municipio (160.977 habitantes) (3).

Tabla 1

Evolución de la población extranjera en Getafe (en número de personas)

\begin{tabular}{cccccc}
\hline 2000 & 2001 & 2002 & 2003 & 2004 & 2005 \\
\hline 3.252 & 8.181 & 11.638 & 13.310 & 15.936 & 16.314 \\
\hline
\end{tabular}


Más allá de esta evolución ascendente de la población extranjera en nuestro país hay que tener en cuenta que la inmigración pone a prueba los recursos de los sujetos para afrontar la dura tarea de asentamiento en un país desconocido. La situación de inmigración implica en muchas ocasiones marginación social, escasos recursos económicos, falta de redes de apoyo social y desconocimiento de los recursos y dispositivos existentes en el país de acogida en materia social y sanitaria $(4,5)$. Los problemas de Salud Mental constituyen uno de los principales motivos de consulta y de morbilidad prevalente de este colectivo en los servicios sanitarios de la Comunidad de Madrid (6).

El crecimiento del fenómeno de la inmigración en nuestro país, a pesar de ser muy rápido en los últimos años, es relativamente reciente. Por este motivo aún no existen suficientes estudios sobre la utilización de los servicios de salud mental por parte de los inmigrantes. La mayoría de estudios realizados en este sentido (acceso y utilización de servicios psiquiátricos por parte de la población inmigrante) procede de otros países, fundamentalmente EE. UU., Reino Unido y países nórdicos (7). Con este trabajo, se pretende observar la adhesión al tratamiento, así como las características sociodemográficas y psicopatológicas de la población inmigrante que acude a un Centro de Salud Mental y que pueden mediar en sus dificultades para cumplimentar los tratamientos.

\section{Método}

Se realizó un estudio con todos los pacientes del colectivo de inmigrantes que asistieron a los Centros de Salud Mental del Área 10 durante un periodo de tres meses (desde el 1 de abril hasta el 30 de junio de 2004): un total de 95 sujetos (51 correspondientes al CSM de Getafe y 44 al CSM de Parla). Se cumplimentó un protocolo con cada paciente que recogía información acerca de los siguientes factores: edad, nacionalidad de origen, tiempo de residencia, dominio del castellano, diagnóstico (agrupaciones diagnósticas CIE-9), y adhesión al tratamiento. Esta última variable se evaluó estudiando el porcentaje de asistencia a las entrevistas programadas en los seis meses siguientes a la inclusión del paciente en el estudio (adhesión al tratamiento $=n .^{\circ}$ de citas a las que acude el paciente $/ \mathrm{n} .^{\circ}$ total de citas programadas). Los resultados obtenidos se compararon con los proporcionados por un grupo control compuesto por otros 95 pacientes de nacionalidad española. Para el diseño de este grupo control se emparejó a cada paciente inmigrante con el siguiente paciente (a partir del número de historia) de nacionalidad española que hubiera acudido al mismo CSM y que fuera equiparable en cuanto al género, el diagnóstico y el programa en el que había sido atendido. De esta forma se obtuvo una muestra final de 190 
pacientes, las características sociodemográficas del subgrupo de inmigrantes se resumen en la Tabla 2; coincidiendo con las del grupo control en cuanto al género, edad (media de la población infanto-juvenil: 11,53, D.T.: 3,47; media de la población adulta: 34,62 , D.T.: 13,47 ), y programa de atención recibida. Los análisis se llevaron a cabo con la versión 9.0 para Windows del programa SPSS.

Tabla 2

Grupo de inmigrantes $(n=95)$

\begin{tabular}{|c|c|c|c|c|}
\hline Género & \multicolumn{2}{|l|}{ Varones: $41,1 \%$} & \multicolumn{2}{|c|}{ Mujeres: $58,9 \%$} \\
\hline Programa & \multicolumn{2}{|l|}{ Infantil: $25,3 \%$} & \multicolumn{2}{|c|}{ Adultos: $74,7 \%$} \\
\hline Intervención & \multicolumn{2}{|c|}{ Casos nuevos: $66,3 \%$} & \multicolumn{2}{|c|}{ Revisiones: $33,7 \%$} \\
\hline \multirow{2}{*}{ Edad } & 0-16 años: $27,1 \%$ & \multicolumn{2}{|c|}{ 17-25 años: $18,8 \%$} & 26-40 años: $31,8 \%$ \\
\hline & 41-64 años: $21,2 \%$ & \multicolumn{2}{|c|}{$>65$ años: $1,2 \%$} & \\
\hline \multirow{2}{*}{ Procedencia } & Europa Este: $8,4 \%$ & \multicolumn{2}{|c|}{ Resto Europa: $10,5 \%$} & Magrebíes: $24,2 \%$ \\
\hline & Subsaharianos: $5,3 \%$ & \multicolumn{2}{|c|}{ Asia: $2,1 \%$} & América C y S: 49,5\% \\
\hline \multirow{2}{*}{ Tipo de inmigración } & \multicolumn{2}{|c|}{ Económico: $73,9 \%$} & \multicolumn{2}{|c|}{ Refugiado: $3,3 \%$} \\
\hline & \multicolumn{2}{|c|}{ Establecido: $13 \%$} & \multicolumn{2}{|c|}{ Otros: $9,8 \%$} \\
\hline \multirow{2}{*}{ Nivel de español } & \multicolumn{2}{|l|}{ Bien: $74,2 \%$} & \multicolumn{2}{|c|}{ Mal: $12,9 \%$} \\
\hline & \multicolumn{2}{|l|}{ Regular: $9,7 \%$} & \multicolumn{2}{|c|}{ No habla: $3,2 \%$} \\
\hline T. residencia & \multicolumn{4}{|c|}{ Media: 6,08 años; Desviación Típica: 5,03 años } \\
\hline
\end{tabular}

\section{Resultados}

En primer lugar, se aplicó una prueba t para estudiar las diferencias entre ambos grupos en cuanto a su asistencia al centro. La media de adhesión al tratamiento para el grupo de inmigrantes fue de 75,56 mientras que en el grupo control fue de 88,43 hallándose una diferencia media estadísticamente significativa $(t=-4,53 ; p=0,000)$ entre ambos grupos de 15,05. En la Tabla 3 se recoge el tanto por ciento de asistencia a las citas programadas durante el periodo seleccionado tanto en la población de inmigrantes como en el grupo control de población española.

Una vez comprobadas las diferencias entre la muestra de inmigrantes y el control en cuanto a la adhesión al tratamiento, el análisis estadístico se centró en el grupo de inmigrantes para estudiar qué características podían estar relacionadas con su menor porcentaje de asistencia. 
Tabla 3

Porcentajes de asistencia al CSM

\begin{tabular}{ccc}
\hline & Grupo de inmigrantes & Grupo de españoles \\
\hline Acuden a todas las citas & $34,7 \%$ & $57,9 \%$ \\
\hline $\begin{array}{c}\text { Acuden entre el 75\% } \\
\text { y el 99\% de las citas }\end{array}$ & $20,0 \%$ & $25,3 \%$ \\
\hline $\begin{array}{c}\text { Acuden entre el 50\% } \\
\text { y el 74\% de las citas }\end{array}$ & $35,8 \%$ & $13,7 \%$ \\
\hline $\begin{array}{c}\text { Acuden entre el 25\% } \\
\text { y el 49\% de las citas }\end{array}$ & $3,2 \%$ & $1,1 \%$ \\
\hline $\begin{array}{c}\text { Acuden a menos del } \\
24 \% \text { de las citas }\end{array}$ & $6,3 \%$ & $2,1 \%$ \\
\hline
\end{tabular}

En primer lugar, se realizó una comparación entre los lugares de procedencia de los inmigrantes respecto a su índice asistencial al centro mediante un ANOVA, con el objeto de estudiar si existían diferencias en adhesión al tratamiento en función del lugar de origen del inmigrante (Figura 1), no encontrándose significación estadística $(\mathrm{F}=1,548 ; \mathrm{p}=0,183)$. Tampoco se encontraron diferencias estadísticamente significativas $(\mathrm{F}=1,270 ; \mathrm{p}=0,270)$ al estudiar la adhesión al tratamiento en función del diagnóstico del paciente (Figura 2), ni entre los casos nuevos que acudían por primera vez al centro (evaluaciones) y los casos de seguimiento $(\mathrm{F}=0,177 ; \mathrm{p}=0,675)$.

Figura 1

Lugar de origen del inmigrante

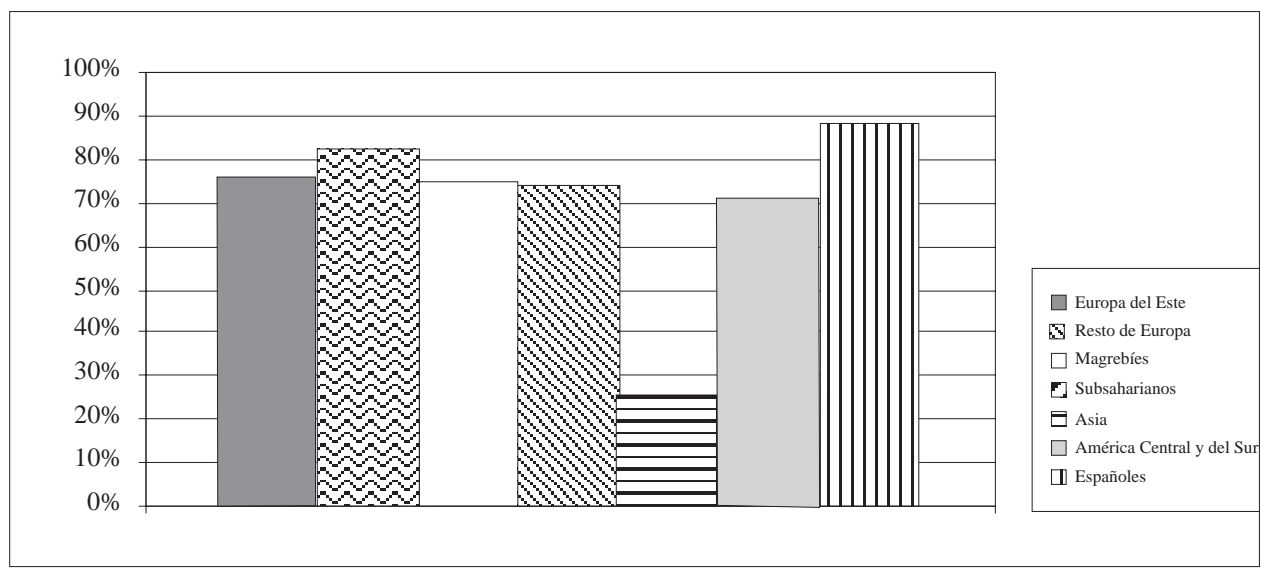


Figura 2

Distribución de los diagnósticos en la muestra

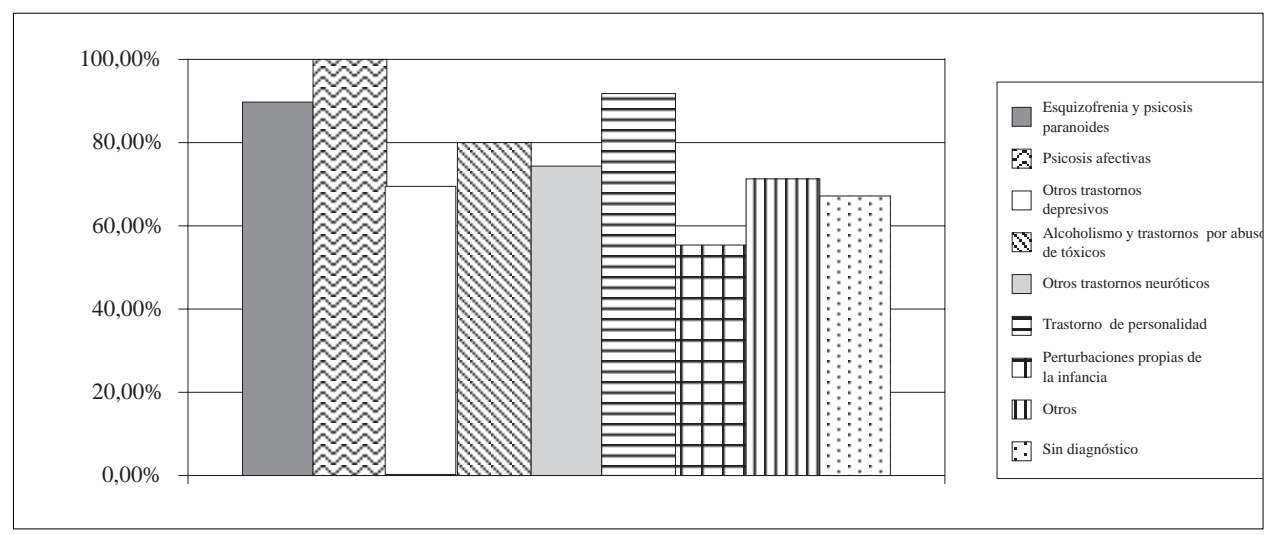

Asimismo tampoco se encontraron diferencias estadísticamente significativas al analizar las desigualdades en adhesión al tratamiento en función del sexo y la edad. En cualquier caso, en cuanto al sexo, se obtuvo un porcentaje más alto de asistencia en el grupo de varones (Tabla 4). Al estudiar la edad, se observó una tendencia hacia una mayor adhesión al tratamiento a medida que aumentaba la edad de los cohortes etarios, excepto en el grupo de adultos jóvenes -entre 26 y 40 años- (Tabla 5). A pesar de esta tendencia tampoco se encontraron diferencias significativas al comparar los porcentajes de asistencia entre el Programa InfantoJuvenil y el de Adultos (Tabla 6).

Tabla 4

Adhesión al tratamiento en función del sexo $(F=0,003 ; p=0,959)$

\begin{tabular}{cccc}
\hline & N & Media & D.T. \\
\hline Mujeres & 56 & 68,33 & 3,71 \\
\hline Varones & 39 & 78,64 & 4,40 \\
\hline
\end{tabular}


Tabla 5

Adhesión al tratamiento en función de la edad ( $F=2,142 ; p=0,083)$

\begin{tabular}{lccc}
\hline & $\mathrm{N}$ & Media & D.T. \\
\hline $0-16$ años & 23 & 66,39 & 5,95 \\
\hline $17-25$ años & 16 & 84,35 & 4,94 \\
\hline $26-40$ años & 27 & 65,04 & 6,08 \\
\hline $41-64$ años & 18 & 80,87 & 4,46 \\
\hline$>65$ años & 1 & 83,33 & - \\
\hline
\end{tabular}

Tabla 6

Adhesión al tratamiento en función del Programa $(F=1,229 ; p=0,270)$

\begin{tabular}{cccc}
\hline & $\mathrm{N}$ & Media & D.T. \\
\hline $\begin{array}{c}\text { Programa } \\
\text { Infanto-juvenil }\end{array}$ & 24 & 67,09 & 28,95 \\
\hline Programa Adultos & 71 & 74,42 & 27,63 \\
\hline
\end{tabular}

Por último, tampoco se encontró significación estadística al estudiar la adhesión al tratamiento en el grupo de inmigrantes en función de variables relacionadas con su establecimiento en España. Se realizó un análisis de regresión lineal para estimar la relación existente entre la variable adhesión y el tiempo de residencia en España (medido en n. $^{\circ}$ de meses), como se aprecia en la Figura 3. La correlación obtenida fue: $r=0,004$ (varianza $=28,515$ ). Además se encontró una relación inversa entre el dominio del idioma español y la adhesión al tratamiento, de forma que los pacientes más cumplidores con las citas eran aquellos que dominaban menos el idioma (tabla 7).

Tabla 7

Adhesión al tratamiento en función del dominio del idioma $(\mathrm{F}=2,025 ; \mathrm{p}=0,116)$

\begin{tabular}{lrrc}
\hline & N & Media & D.T. \\
\hline Habla bien español & 69 & 71,73 & 28,74 \\
\hline Habla regular el español & 9 & 57,17 & 25,31 \\
\hline Habla mal el español & 12 & 85,80 & 21,02 \\
\hline No habla nada de español & 3 & 83,33 & 28,87 \\
\hline
\end{tabular}


Figura 3

Relación entre la variable adhesión y el tiempo de residencia en España (n. ${ }^{o}$ meses).

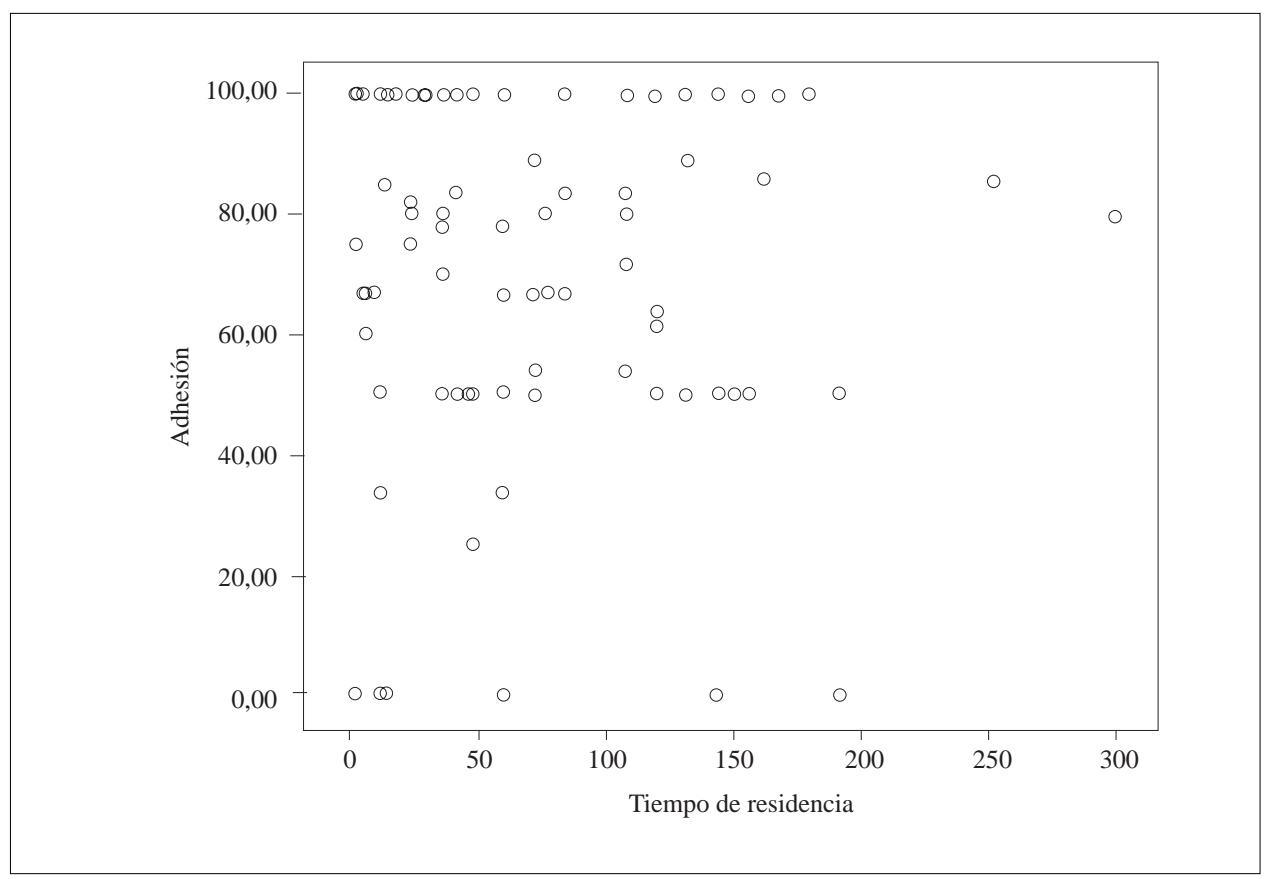

\section{Discusión}

La población extranjera se ha incrementado de forma llamativa en España en los últimos años. Este vertiginoso ascenso del fenómeno de las migraciones ha conllevado un incremento en la atención a inmigrantes en los Servicios de Salud Mental, a la vez que se observan características particulares de este colectivo, muy especialmente en cuanto a la adhesión al tratamiento en comparación con otros pacientes de nacionalidad española. Existen numerosos estudios que han observado que ciertos grupos de inmigrantes tienen mayor tendencia que los nativos a abandonar el tratamiento psicológico y psiquiátrico, sosteniendo la hipótesis de que los pacientes inmigrantes siguen itinerarios más negativos en el acceso a los servicios de salud mental que los ciudadanos nativos (7). Por «itinerario negativo» se entiende que la forma de acceso a los servicios de salud mental por parte de la población inmigrante es menos deseable que la de la población nativa, implicando con mayor frecuencia la intervención de la policía o el ingreso judicial e involuntario. 
Sin embargo, a pesar del citado aumento de población extranjera en nuestro país, el incremento en la asistencia del colectivo de inmigrantes a los Servicios de Salud Mental no ha sido tan elevado como para que se equiparen las cifras de utilización de estos dispositivos con las observadas en la población española. Por ejemplo, en los tres meses estudiados en este trabajo el número total de pacientes inmigrantes atendidos en el Centro de Salud Mental de Getafe fue de 51, lo que supone tan sólo un $0,32 \%$ de la población inmigrante del municipio de Getafe en 2004 (15.936 habitantes); en comparación con el 0,85\% de prevalencia en la población total. A partir de la Ley 4/2000 de 11 de enero sobre derechos y libertades de los extranjeros en España y su posterior reforma por la Ley 8/2000 del mismo año tienen derecho a la obtención de la Tarjeta Sanitaria, que garantiza la asistencia sanitaria pública en las mismas condiciones que los españoles, todos los extranjeros que se encuentren empadronados (8). A pesar de ello todavía hay muchas personas inmigrantes en situación de irregularidad administrativa que por miedo o desconocimiento no están empadronados y, por tanto, carecen de asistencia sanitaria normalizada. Asimismo, hay que tener en cuenta que incluso entre aquellos que se encuentran en situación regularizada, al estudiar el acceso de este colectivo a los servicios sanitarios debemos considerar otros factores sociales y culturales. Las personas inmigrantes, en general, suelen priorizar trabajo frente a salud y sobre todo en los primeros momentos del proceso migratorio, sólo acuden a los servicios sanitarios cuando se encuentran mal buscando una solución inmediata a sus problemas, lo que lleva a una mayor saturación de los Servicios de Urgencias y un mal uso de los dispositivos de atención ambulatoria. En esta línea, algunos autores han encontrado que los inmigrantes tardan más tiempo que los sujetos de la población general en acudir a los Servicios de Salud Mental ante un primer episodio psicótico (9).

Estos hallazgos contrastan con el postulado tradicional de que los inmigrantes tienen tasas de ingreso psiquiátrico más elevadas que los nativos y que supone que el colectivo de inmigrantes tiene tasas más elevadas de algunos trastornos mentales como la esquizofrenia (10). Más allá de las posibles diferencias entre los distintos dispositivos de asistencias, esta aparente contradicción podría indicar la difícil generalización de los resultados sobre los problemas de salud mental en los inmigrantes, y la importancia de realizar estudios específicos para la población del país.

Pero a la vista de nuestros datos, el único problema no es que las personas inmigrantes acudan poco a la consulta de Salud Mental, sino que además aquellos que vienen muestran una menor adhesión al tratamiento. En otro trabajo con población inmigrante en la Comunidad de Madrid, Gotor y González-Juárez (2004) (11) han demostrado recientemente que la continuidad de los cuidados de salud mental es menor en los inmigrantes que ingresan en las unidades de hospi- 
talización. En nuestro estudio observamos que el porcentaje de asistencia a las citas en la población de inmigrantes es menor que la población de españoles, alcanzando estas diferencias significación estadística. La explicación de este hallazgo puede estar mediada por diversos factores clínicos y culturales que pueden influir en la relación terapéutica, la adhesión al tratamiento en Salud Mental y los abandonos.

Una primera hipótesis a tener en cuenta señala la destreza del idioma como un elemento relevante a la hora de justificar la peor cumplimentación del tratamiento por parte del colectivo de inmigrantes. Pero, paradójicamente, los datos que proporcionan nuestro estudio sugieren una relación inversa entre el dominio del idioma español y la adhesión al tratamiento. De hecho, el porcentaje más bajo de adhesión al tratamiento fue el del grupo de inmigrantes que mejor dominaban el castellano (habla regular y bien el español). Una posible explicación a este fenómeno podría ser que los pacientes que no conocen el idioma sólo acuden a los Servicios de Salud Mental en caso de patologías más graves, presentando índices de adhesión al tratamiento más elevados que los pacientes que dominan el idioma y que, en muchos casos, demandarían asistencia psicológica y psiquiátrica con problemáticas más leves. El hecho de que al estudiar la asistencia a las citas en función del diagnóstico clínico se encuentre un porcentaje de adhesión más alto entre las patologías más graves (esquizofrenia, psicosis afectivas y trastornos de personalidad) apoyaría también este argumento $(12 ; 13)$.

Otro factor relacionado directamente con la destreza del idioma es el lugar de origen del inmigrante y el tiempo que lleva establecido en nuestro país. Sin embargo, tampoco se encontraron diferencias en función de los países de procedencia de los sujetos y el tiempo transcurrido desde la inmigración. De todas las regiones geográficas analizadas es el subgrupo de pacientes de origen asiático el que presenta un porcentaje de asistencia más bajo, lo que hace pensar en la inevitable barrera cultural con la que se encuentra este colectivo a la hora de acudir a los servicios sanitarios por algún problema psíquico. A este dato hay que añadir el hecho de que es precisamente este subgrupo el que menos demanda asistencia profesional en los Servicios de Salud Mental (representa únicamente un 2,1\% de la muestra). En el polo opuesto se encontrarían los pacientes de origen europeo que constituyen el grupo más cumplidor en cuanto a la asistencia a las citas en nuestro centro, lo que sugiere que entre este colectivo la barrera cultural es menos pronunciada que en el anterior y que probablemente en sus países de origen exista más tradición sobre el uso de dispositivos relacionados con el tratamiento psicológico y psiquiátrico.

Otros trabajos que también han encontrado una infrautilización de los Servicios de Salud Mental por parte de los inmigrantes han propuesto otras posibles explicaciones para este fenómeno. Por ejemplo, algunos autores han halla- 
do que grupos como los afroamericanos son más reticentes al uso de Servicios de Salud Mental debido a que tienen miedo al tratamiento y a un posible ingreso, e incluso en algunos trabajos se ha encontrado que los varones de raza negra tienen mayor probabilidad de ser enviados a una institución penitenciaria que a una psiquiátrica $(14 ; 15)$. También se ha observado que en algunos colectivos de inmigrantes las redes de apoyo informal constituyen la fuente principal de apoyo social en detrimento de los programas comunitarios y los servicios más formales (16).

En conclusión, a la vista de los resultados es evidente que las diferencias culturales y las condiciones de vida y trabajo de muchas de las personas inmigrantes dificultan el acceso y la utilización por parte de este colectivo de los dispositivos de Salud Mental. La edad de migrar y los motivos para hacerlo, las circunstancias en que tiene lugar la migración, las diferencias entre el lugar de origen y el de llegada, la actitud mutua entre los grupos de emigrantes y la sociedad huésped y las diferencias en la incidencia y prevalencia de problemas de salud mental en las poblaciones son, todas ellas, variables que determinan esta complejidad (17). No es posible explicar esta situación apelando a factores aislados como el dominio del idioma, el lugar de origen, el tiempo de establecimiento en nuestro país, la edad o el diagnóstico clínico, por lo que parece imprescindible un abordaje complejo y multicausal del fenómeno, insistiendo en la fase de acogida y los primeros contactos del paciente con los dispositivos para fomentar una mayor adhesión al tratamiento.

\section{BIBLIOGRAFÍA}

(1) Ministerio del Interior. Balance 2005. http://www.mir.es/oris.

(2) INE. Padrón Municipal 2005. http://www.ine.es.

(3) Grupo de trabajo de Intervención Social del Plan Local de Inmigración del Ayuntamiento de Getafe, Informe sobre la intervención social en el ámbito de la inmigración en la ciudad de Getafe, Madrid, Ayuntamiento de Getafe, 2005.

(4) DíAz, M., Apoyo social y salud mental en inmigrantes: sus efectos sobre la integración, Madrid, Consejería de Servicios Sociales, 2003.

(5) SANZ, B.; CAStillo, S., «La salud de la población inmigrante», en MAZARRASA-GERMÁNSÁnchez, M., Salud Publica y Enfermería Comunitarias, Madrid, Mc Graw-Hill Interamericana, 2003.

(6) GimÉnEZ, C., Inmigrantes extranjeros en Madrid, Madrid, Consejería de Integración Social, 1993.

(7) BaCA-García, E.; PéRez, M., y otros, «Acceso, itinerarios y utilización de los servicios psiquiátricos en la población inmigrante», Monografías de Psiquiatría, 2005, 4, pp. 11-17.

(8) Ley Orgánica 8/2000, Reforma de la 4/2000 sobre Derechos y Libertades de los Extranjeros en España y su integración Social (dic. 22, 2000). 
(9) Harrison, G.; Holton, A.; Nelson, D., «Severe Mental Disorder in Afro-Caribbean Patients: Some Social, Demographic and Service Factors», Psychological Medicine, 1989, 19, pp. 683-696.

(10) King, M.; CoKer, E.; Leavy, G., y otros, «Incidence of Psychotic Illness in London: a Comparison of Ethnic Groups», British Medical Journal, 1994, 309, pp. 1115-1119.

(11) Gotor, L.; GonZÁLEZ-Jú́ReZ, C., «Psychiatric Hospitalization and Continuity of Care in Inmigrants Treated in Madrid», Social Psychiatry and Pychiatric Epidemiology, 2004, 39, pp. 560-568.

(12) McGrath, J.; El-SAadi, O.; CARdy, S., y otros, «Urban Birth and Migrant Status as Risk Factor for Psychosis: an Australian Case-Control Study», Soc. Psychiatry Psychiatr. Epidemiol, 2001, 36, pp. 533-536.

(13) Burguillo, F., y otros, «Patoplastia en inmigración», Archivos de Psiquiatría, 2003, 66, pp. 305-314.

(14) Cohen, R.; Parmelee, D.; Irwin, L., «Characteristics of Children in a Psychiatric Hospital and a Corrections Facility», Journal of American Academy of Child and Adolescent Psychiatry, 1990, 29, pp. 909-913.

(15) Sussman, L. K.; RobBins, L. N.; EARLs, F., «Treatment Seeking for Depression by Black and White Americans», Social Science and Medicine, 1987, 24, pp.187-196.

(16) Hernández, S.; Pozo, C.; Alonso, E., «The Role of Informal Social Support in Needs Assesment: Proposal and Application of a Model to Asses Inmigrants Needs in the South of Spain», Journal of Community and Applied Social Psychology, 2004, 14, pp. 284-298.

(17) Alda, M.; Moles, B.; GarCía CAMPAYO, J., «Salud Mental e inmigración», Monografías de Psiquiatría, 2005, 4, pp. 2-10.

* Luis Javier Sanz Rodríguez, psicólogo Clínico; Isabel Elustondo Ferre, psiquiatra; Magdalena Valverde Gómez, psiquiatra; José F. Montilla García, psiquiatra, jefe del Servicio de Psiquiatría y Salud Mental; Marta Miralles de Imperial Mora-Figueroa, trabajadora Social. Servicio de Psiquiatría y Salud Mental del Área 10. Getafe (Madrid).

Correspondencia: Luis Javier Sanz Rodríguez. C/ Mayor, 23. 28991 Getafe (Madrid). ljsanz@ cop.es

** Fecha de recepción: 5-II-2007. 